Dr Yoanna Skrobik

\title{
Chasing the elusive notion of delirium causality
}

Received: 5 October 2015

Accepted: 8 October 2015

Published online: 16 October 2015

(C) Springer-Verlag Berlin Heidelberg and ESICM 2015

Dr Y. Skrobik (

Department of Medicine, McGill University Health Center, 1001 Decarie Boulevard, Montréal, QC H4A 3J1, Canada

e-mail: yoanna.skrobik@mcgill.ca

Delirium and its potential association with modifiable critical care practices have much preoccupied clinicians and researchers over the past two decades. Early mobility clearly decreases delirium incidence and duration. Mobilization remains sadly under-utilized in a culture where belief drives sedation for the sake of 'patient safety', despite growing evidence that sedation is anything but safe. Convictions as to delirium causality are currently disproportionate to the available evidence, given the complex interplay between patient fragility, acuity of illness and its related biology, and iatrogenic factors. The question of whether sedation, and more specifically sedative molecule choices, influences delirium occurrence, duration, and severity remains the subject of enthusiastic debate.

A recent publication by Zaal and colleagues [1] reports on a retrospective cohort observational study of critically ill adults, with the goal of testing whether benzodiazepine exposure is a risk factor for transition from an awake state without delirium to delirium. Of the 1112 patients observed in the study, the majority (814) were exposed to benzodiazepines for a mean duration of 3 days (and $48 \%$ of the observation days); delirium occurred in $48 \%$ of the patient cohort on $27 \%$ of the observation days.
The authors analyzed the odds ratio for the transition targeted by their primary question (awake to delirious and awake) using Markov modeling [2]. This methodology is particularly useful in addressing a clinical risk that occurs, and may recur, over time, as is the case with delirium. In addition, back-and-forth transitions (Fig. E1 in the Zaal article) from one state to the other can be considered, which reflects our clinical reality. In addition to the primary analysis, all transitions in the categories of awake-no delirium, delirium, coma, discharged and dead were measured. Importantly, and in keeping with the Markov property of the analysis, duration of benzodiazepine administration prior to the transition was not taken into account. Covariables included in the multivariable analysis were taken from a recent systematic review of delirium risk factors by the same group [3]. Excessive alcohol consumption and dementia, considered to increase the risk of delirium [3], were not included because of the low number of representative patients in this single center, limiting the generalizability of the conclusion in populations where these risk factors are prevalent.

The primary outcome result, an odds ratio of transitioning from awake without delirium towards delirium of 1.04 [95\% confidence interval (CI): 1.03-1.05] per $5 \mathrm{mg}$ midazolam administered, led the authors to conclude that benzodiazepines are associated with an increased risk of delirium the following day in wakeful patients. However, an analysis of benzodiazepines administered in bolus form vs. as an infusion was also performed, and serves as a representative portrayal of 'real-life' ICU practice patterns. Patients could have received both infusions and boluses on the same day; infusions represented $19 \%$ of patient-days [with median doses of $99 \mathrm{mg}$, interquartile ratio (IQR): 29.4-212] and the boluses $41 \%$, with a median dose of 4.1 mg, IQR: 1.9-8.3. In awake, delirium-free benzodiazepine infusion recipients, the odds for delirium the next day (per $5 \mathrm{mg}$ midazolam administered) were nearly identical to those described in the primary (overall) outcome 
analysis. Benzodiazepine boluses, however, were not associated with delirium the next day. Similarly, presence of delirium on the 2 nd day after benzodiazepine exposure was only associated with benzodiazepine infusions. Interestingly, transitions from coma to delirium were also not associated with the administration of benzodiazepines.

This paper raises a number of interesting questions. The authors acknowledge that not incorporating factors such as renal function, co-administered drugs metabolized through the same cytochrome CYP-450 pathway known to affect sedation and benzodiazepine serum levels [4], age, and other determinants of midazolam and other benzodiazepine clearance in the analysis made their assessment of 'true' benzodiazepine exposure challenging. All efforts were made to assess delirium when the patients were most wakeful, but, as the discussion highlights, sedation-associated positive delirium scores may have occurred [5]. Moreover, the apparent discordance between Zaal et al.'s findings and previously published data correlating higher midazolam serum levels with lower incidences of delirium [4] remains unexplained. Why bolus vs. intermittent administration should differ in this respect is perplexing unless one invokes duration and dose that lengthen effect incrementally over time, since the pharmacokinetic properties with continuous vs. bolus midazolam administration in the critically ill are similar [6]. Biological sample-based exposure assessments, available largely in critically ill children, show that the determinants of serum and tissue concentration are highly variable [7] and influenced by co-administered competitively interactive drugs $[4,8]$. This could theoretically account for highly variable 'true' exposure in this cohort (and paradoxical results) if the variability in metabolism were disproportionate to the large number of patients and the range of drug doses administered. If this were the case, the drug exposure association might be confounded by tissue and/or serum concentrations of parent drug and bioactive metabolite determinants.

This is a challenging area to study. Markov modeling — and, indeed, most statistical analyses - are based on the premise of firmly established and contained states. Delirium does not lend itself easily to being so unequivocally framed. In general terms, diagnostic criteria and structured interviews reduce the well-established unreliability inherent to any psychiatric diagnosis [9]. Observational studies conducted in the ICU necessarily include mechanically ventilated patients (78\% here), making interviews challenging. Investigators must thus rely on imperfect 'delirium' diagnostic surrogates with screening tools such as CAM-ICU, for which confounding by sedation and the widely varying 'incidence' rate across ICU populations are well established [10].

Zaal et al.'s paper offers much opportunity for reflection. The acquisition costs [11] and familiar complication rates of benzodiazepines make them an attractive choice as a sedative. With their use, maintenance of the light sedation endorsed by Zaal and colleagues can be challenging, since both the parent drug and the active metabolite accumulate; this effect, and the excessive sedation associated with it, may be particularly relevant with higher doses and with infusions. This large observational trial should invite caution on the part of practitioners whose practice incorporates benzodiazepine infusions. It is also likely to stimulate academics, clinicians, and researchers in their debate revolving around the association between benzodiazepines and delirium.

\section{Compliance with ethical standards}

Conflict of interest The author has no conflicts to declare.

\section{References}

1. Zaal IJ, Devlin JW, Hazelbag M, Klein Klouwenberg PMC, van der Kooi AW, Ong DSY, Cremer OL, Groenwold RH, Slooter AJC (2015) Benzodiazepineassociated delirium in critically ill adults. Intensive Care Med. doi: 10.1007/s00134-015-4063-z

2. Sonnenberg FA, Beck JR (1993) Markov models in medical decision making. Med Decis Making 13:322-338

3. Zaal IJ et al (2015) A systematic review of risk factors for delirium in the ICU. Crit Care Med 43(1):40-47

4. Skrobik et al (2013) Predisposing factors to coma and delirium: fentanyl and midazolam exposure, CYP3A5, $\mathrm{ABCB} 1$ and $\mathrm{ABCG} 2$ genetic polymorphisms, and inflammatory factors. Crit Care Med 41(4):999-1008
5. Patel SB, Poston JT, Pohlman A et al (2014) Rapidly reversible, sedationrelated delirium versus persistent delirium in the intensive care unit. Am J Respir Crit Care Med 189:658-665

6. Beigmohammadi MT et al (2013) Pharmacokinetics alterations of midazolam infusion versus bolus administration in mechanically ventilated critically ill patients. Iranian J Pharmaceut Res 12(2):483-488

7. Ince I et al (2012) Critical illness is a major determinant of midazolam clearance in children aged 1 month to 17 years. Ther Drug Monit 34:381-389

8. Spriet I et al (2009) Mini-series: II. clinical aspects. clinically relevant CYP450-mediated drug interactions in the ICU. Intensive Care Med 35:603-612
9. Aboraya A et al (2006) The reliability of psychiatric diagnosis revisited: the clinician's guide to improve the reliability of psychiatric diagnosis. Psychiatry 3:41-50

10. The Can delirium Assessments Be Accurately Labelled (CABAL) Investigators group, Devlin JW et al (2013) The accurate recognition of delirium in the ICU: the emperor's new clothes? Intensive Care Med 39:2196-2199

11. Wunsch H (2012) Weighing the costs and benefits of a sedative. JAMA 307(11):1195-1197 